\title{
Discriminative Feature Selection via Multiclass Variable Memory Markov Model
}

\author{
Noam Slonim \\ School of Engineering and Computer Science and Interdisciplinary Center for Neural Computation, \\ The Hebrew University of Jerusalem, Jerusalem 91904, Israel \\ Email:noamm@cs.huji.ac.il

\section{Gill Bejerano} \\ School of Engineering and Computer Science, The Hebrew University of Jerusalem, Jerusalem 91904, Israel
} Email: jill@cs.huji.ac.il

\author{
Shai Fine \\ IBM Research Laboratory in Haifa, Haifa University, Mount Carmel, Haifa 31905, Israel \\ Email:fshai@il.ibm.com
}

\section{Naftali Tishby}

School of Engineering and Computer Science and Interdisciplinary Center for Neural Computation, The Hebrew University of Jerusalem, Jerusalem 91904, Israel

Email: tishby@cs.huji.ac.il

Received 18 April 2002 and in revised form 15 November 2002

\begin{abstract}
We propose a novel feature selection method based on a variable memory Markov (VMM) model. The VMM was originally proposed as a generative model trying to preserve the original source statistics from training data. We extend this technique to simultaneously handle several sources, and further apply a new criterion to prune out nondiscriminative features out of the model. This results in a multiclass discriminative VMM (DVMM), which is highly efficient, scaling linearly with data size. Moreover, we suggest a natural scheme to sort the remaining features based on their discriminative power with respect to the sources at hand. We demonstrate the utility of our method for text and protein classification tasks.
\end{abstract}

Keywords and phrases: variable memory Markov (VMM) model, feature selection, multiclass discriminative analysis.

\section{INTRODUCTION}

Feature selection is one of the most fundamental problems in pattern recognition and machine learning. In this approach, one wishes to sort all possible features using some predefined criteria and select only the "best" ones for the task at hand. It thus may be possible to significantly reduce model dimensions without impeding the performance of the learning algorithm. In some cases one may even gain in generalization power by filtering irrelevant features (cf. [1]). The need for a good feature selection technique also stem from the practical concern that estimating the joint distribution (between the classes and the feature vectors), when either the dimensionality of the feature space or the number of classes is very large, requires impractical large training sets. Indeed, increasing the number of features while keeping the number of samples fixed can actually lead to decrease in the accuracy of the classifier $[2,3]$.
In this paper, we present a novel method for feature selection based on a variable memory Markov (VMM) model [4]. For a large variety of sequential data, statistical correlations decrease rapidly with the distance between symbols in the sequence. In particular, consider the conditional (empirical) probability distribution on the next symbol given its preceding subsequence. If the statistical correlations are indeed decreasing, then there exists a length $L$ (the memory length) such that the above conditional probability does not change substantially if conditioned on subsequences longer than $L$. This suggests modeling the sequences by Markov chains of order $L$. However, such models grow exponentially with $L$ which makes them impractical for many applications. One elegant solution to this problem was proposed by Ron et al. [4]. The underlying observation in that work was the fact that in many natural sequences, the memory length depends on the context and thus it is not fixed. Therefore, 


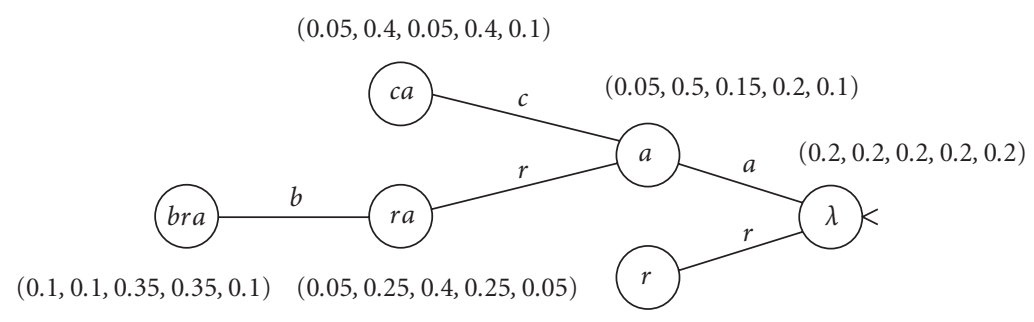

$(0.6,0.1,0.1,0.1,0.1)$

Figure 1: An example of a PST. The root node corresponds to the empty suffix, the nodes in the first level correspond to suffixes of order one, and so forth. The string inside each node is a memorized suffix and the adjacent vector is its probability distribution over the next symbol of the alphabet $\Sigma=\{a, b, c, d, r\}$. For example, the probability to observe $c$ after the substring bara, whose largest suffix in the tree is $r a$, is $P(c \mid$ bara $)=P_{r a}(c)=0.4$. Similarly, since suff $($ bacara $)=r a$, the probabilities $P(\sigma \mid$ bacara $)=\{0.05,0.25,0.4,0.25,0.05\}$ for $\sigma \in\{a, b, c, d, r\}$, respectively.

Ron et al. introduced a learning algorithm using a construction called prediction suffix tree (PST), which preserves the minimal subsequences (of variable lengths) that are necessary for precise modeling of the given statistical source (see Figure 1).

While the motivation of Ron et al. was to provide generative statistical modeling for a single source, the current work uses the PST construction to address supervised discrimination tasks. Thus, our first step is to extend the original generative VMM modeling technique to handle several sources simultaneously. Next, since we wish to use the resulting multiclass model to classify new (test) sequences, we are less concerned with preserving source statistics. Rather, we focus on identifying variable length dependencies, that can serve as good discriminative features between the learned categories. This results in a new algorithm, termed discriminative VMM (DVMM).

Our feature selection scheme is based on maximizing conditional mutual information (MI). More precisely, for any subsequence $s$ we estimate the information between the next symbol in the sequence and each statistical source $c \in C$, given that subsequence (or suffix) $s$. We use this estimate as a new measure for pruning less discriminative features out of the model. This yields a criterion which is very different from the one used by the original generative VMM model. In particular, many features may be important for good modeling of each source independently although they provide minor discrimination power. These features are pruned in the DVMM, resulting in a much more compact model which still attains high classification accuracy.

We further suggest a natural sorting of the features retained in the DVMM model. This allows an examination of the most discriminative features, often gaining useful insights about the nature of the data.

\subsection{Related work}

The use of MI for feature selection is well known in machine learning realm, though it is usually suggested in the context of "static" rather than stochastic modeling. The original idea may be traced back to Lewis [5]. It is motivated by the fact that when the a priori class uncertainty is given, maximizing the $\mathrm{MI}$ is equivalent to the minimization of the conditional entropy. This in turn links MI maximization and the decrease in classification error, ${ }^{1}$

$$
H\left(P_{\text {err }}\right)+P_{\text {err }} \log (C-1) \geq H(C \mid X) \geq 2 P_{\text {err }}
$$

where

$$
\begin{gathered}
H(\cdot)=-\sum P(\cdot) \log P(\cdot), \\
H(\cdot \mid \cdot)=-\sum P(\cdot, \cdot) \log P(\cdot \mid \cdot)
\end{gathered}
$$

are the entropy and the conditional entropy, respectively.

Since then, a number of methods have been posed, differing essentially by their method of approximating the joint and marginal distributions, and their direct usage of the mutual information measure (cf. $[8,9,10]$ ). One of the difficulties in applying MI based feature selection methods, is the fact that evaluating the MI measure involves integrating over a dense set, which leads to a computational overload. To circumvent that, Torkkola and Campbell [11] have recently suggested to perform feature transformation (rather than feature selection) to a lower-dimensional space in which the training and analysis of the data is more feasible. Their method is designed to find a linear transformation in the feature space that will maximize the MI between the transformed data and their class labels, and the reduction in computational load is achieved by the use of Renyi's entropy based definition of mutual information which is much more easy to evaluate.

Out of numerous feature selection techniques found in the literature, we would like to point out the work of Della Pietra et al. [12] who devised a feature selection (or rather, induction) mechanism to build $n$-grams of varying lengths, and McCallum's "U-Tree" [13], which build PSTs based on

\footnotetext{
${ }^{1}$ The upper bound is due to Fano's inequality (cf. [6]), and the lower bound can be found, for example, in [7].
} 
the ability to predict the future discounted reward in the context of reinforcement learning.

Another popular approach in language modeling is the use of pruning as a mean for parameter selection from a higher-order $n$-gram backoff ${ }^{2}$ model. One successful pruning criterion, suggested by Stolcke [15], minimizes the "distance" (measured by relative entropy) between the distributions embodied by the original and the pruned models. By relating relative entropy to the relative change in training set perplexity, ${ }^{3}$ a simple pruning criterion is devised, which removes from the model all $n$-grams that change perplexity by less than a threshold. Stolcke shows [15] that in practice this criterion yields a significant reduction in model size without increasing classification error.

A selection criterion, similar to the one we propose here, was suggested by Goodman and Smyth for decision tree design [7]. Their approach chooses the "best" feature at any node in the tree, conditioned on the features previously chosen, and the outcome of evaluating those features. Thus, they suggested a top-down algorithm based on greedy selection of the most informative features. Their algorithm is equivalent to the Shannon-Fano prefix coding, and can also be related to communication problems in noisy channels with side information. For feature selection, Goodman and Smyth noted that with the assumption that all features are known a priori, the decision tree design algorithm will choose the most relevant features for the classification task, and ignore irrelevant ones. Thus, the tree itself yields valuable information on the relative importance of the various features.

A related usage of MI for stochastic modeling is the maximal mutual information (MMI) approach for multiclass model training. This is a discriminative training approach attributed to Bahl et al. [16], designed to directly approximate the posterior probability distribution, in contrast to the indirect approach, via Bayes' formula, of maximum likelihood (ML) training. The MMI method was applied successfully to Hidden Markov Models (HMM) training in speech applications (see, e.g., $[17,18])$. However, MMI training is significantly more expensive than ML training. Unlike ML training, in this approach all models affect the training of every single model through the denominator. In fact this is one reason why the MMI method is considered to be more complex. Another reason is that there are no known easy re-estimation formulas (as in ML). Thus we need to resort to general purpose optimization techniques.

Our approach stems from a similar motivation but it simplifies matters: we begin with a simultaneous ML training for all classes and then select features that maximize the same ob-

\footnotetext{
${ }^{2}$ The backoff recursive rule (cf. [14]) represents $n$-gram conditional probabilities $P\left(w_{n} \mid w_{n-1} \cdots w_{1}\right)$ using $(n-1)$-gram conditional probabilities multiplied by a backoff weight, $\alpha\left(w_{n-1} \cdots w_{1}\right)$, associated with the full history, that is, $P\left(w_{n} \mid w_{n-1} \cdots w_{1}\right)=\alpha\left(w_{n-1} \cdots w_{1}\right) P\left(w_{n} \mid w_{n-1} \cdots w_{2}\right)$, where $\alpha$ is selected such that $\sum P\left(w_{n} \mid w_{n-1} \cdots w_{1}\right)=1$.

${ }^{3}$ Perplexity is the average branching factor of the language model.
}

jective function. While we cannot claim to directly maximize mutual information, we provide a practical approximation which is far less computationally demanding.

\section{VARIABLE MEMORY MARKOV MODELS}

Consider the classification problem to a set of categories $C=\left\{c_{1}, c_{2}, \ldots, c_{|C|}\right\}$. The training data consists of a set of labeled examples for each class. Each sample is a sequence of symbols over some alphabet $\Sigma$. A Bayesian learning framework trains generative models to produce good estimates of class conditioned probabilities, which in turn, upon receiving a new (test) sample $d$, are employed to yield Maximum A Posteriori (MAP) decision rule

$$
\max _{c \in C} P(c \mid d) \propto \max _{c \in C} P(d \mid c) P(c), \quad d \in \Sigma^{*} .
$$

Thus, good estimates of $P(d \mid c)$ are essential for accurate classification. Let $d=\sigma_{1}, \sigma_{2}, \ldots, \sigma_{|d|}, \sigma_{i} \in \Sigma$, and let $s_{i} \in \Sigma^{i-1}$ denote the subsequence of symbols preceding to $\sigma_{i}$, then

$$
P(d \mid c)=\prod_{i=1}^{|d|} P\left(\sigma_{i} \mid \sigma_{1} \sigma_{2} \cdots \sigma_{i-1}, c\right)=\prod_{i=1}^{|d|} P\left(\sigma_{i} \mid s_{i}, c\right) .
$$

Denoting by $\operatorname{suff}\left(s_{i}\right)$ the longest suffix of $s_{i}$, we know that if $P\left(\sigma \mid s_{i}\right)=P\left(\sigma \mid \operatorname{suff}\left(s_{i}\right)\right)$, for every $\sigma \in \Sigma$, then predicting the next symbol using $s_{i}$ is equivalent to a prediction using the shorter context given by $\operatorname{suff}\left(s_{i}\right)$. Thus, in this case it is clear that keeping only $\operatorname{suff}\left(s_{i}\right)$ in the model should suffice for the prediction.

The VMM algorithm [4] aims at building a model which will hold only a minimal set of relevant suffixes. To this end, a suffix tree $\hat{T}$ is built in two steps. First, only suffixes $s \in \Sigma^{*}$ for which the empirical probability in the training data, $\hat{P}(s)$, is nonnegligible, are kept in the model. Thus, rare suffixes are ignored. Next, all suffixes that are not informative for predicting the next symbol are pruned out of the model. Specifically, this is done by thresholding $r \equiv P(\sigma \mid s) / P(\sigma \mid \operatorname{suff}(s))$. If $r \approx 1$ for all $\sigma \in \Sigma$, then predicting the next symbol using $\operatorname{suff}(s)$ is almost identical to using $s$. In such cases $s$ will be pruned out of the model.

\section{MULTICLASS DISCRIMINATIVE VMM}

The VMM algorithm is designed to statistically approximate a single source. A straightforward extension to handle multiclass categorization tasks would build a separate VMM for each class, based solely on its own data, and would classify a new example to the model with the highest score (a oneversus-all approach, e.g., [19]).

Motivated by a generative goal, this approach disregards the possible (dis)similarities between the different categories. Each model aims at best approximating its assigned source. However, in a discriminative framework these interactions may be exploit to our benefit. As a simple example, assume that for some suffix $s$ and every symbol $\sigma \in \Sigma, \hat{P}(\sigma \mid s, c)=$ $\hat{P}(\sigma \mid s)$ for all $c \in C$, that is, the symbols and the categories 


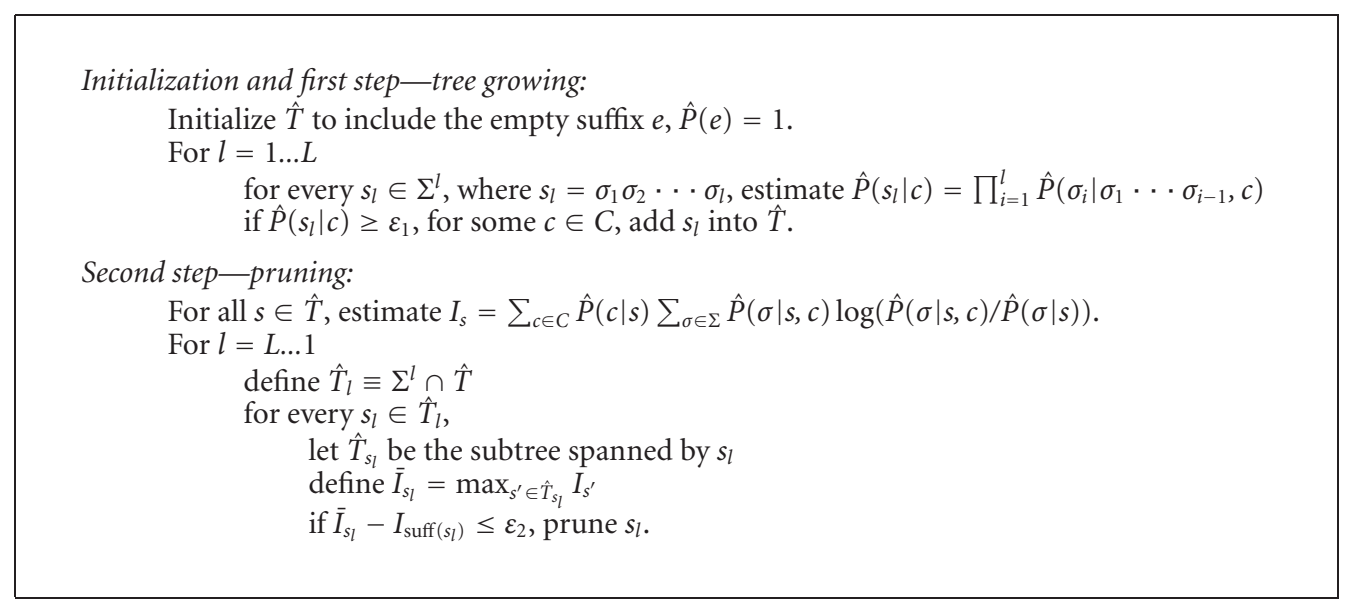

Algorithm 1: Pseudo-code for the DVMM training algorithm.

are independent given s. Since we are only interested in the relative order of the posteriors $\hat{P}(c \mid s)$, these terms may as well be neglected. In other words, preserving $s$ in the model will yield no contribution to the classification task, since this suffix has no discrimination power with respect to the given categories.

We now turn to generalize and quantify this intuition. In general, two random variables $X$ and $Y$ are independent if and only if the MI between them is zero (cf. [6]). For every $s \in \Sigma^{*}$, we consider the following (local) conditional MI,

$$
I_{s} \equiv I(\Sigma ; C \mid s)=\sum_{c \in C} \hat{P}(c \mid s) \sum_{\sigma \in \Sigma} \hat{P}(\sigma \mid c, s) \log \frac{\hat{P}(\sigma \mid c, s)}{\hat{P}(\sigma \mid s)}
$$

where $\hat{P}(c \mid s)$ is estimated using Bayes' formula, $\hat{P}(c \mid s)=$ $\hat{P}(s \mid c) \hat{P}(c) / \hat{P}(s)$, the prior $\hat{P}(c)$ can be estimated by the relative number of training examples labeled with the category $c$, or from domain knowledge, and $\hat{P}(s)=\sum_{c \in C} \hat{P}(c) \hat{P}(s \mid c)$. If $I_{s}=0$, as above, $s$ can certainly be pruned. However, we may define a stronger pruning criterion, which consider also the suffix of $s$. Specifically, if $I_{s}-I_{\text {suff }(s)} \leq \varepsilon_{2}$, where $\varepsilon_{2}$ is some threshold, one may prune $s$ and settle for the shorter memory $\operatorname{suff}(s)$. In other words, this criterion implies that $\operatorname{suff}(s)$ effectively induces more dependency between $\Sigma$ and $C$ than its extension $s$. Thus, preserving $\operatorname{suff}(s)$ in the model should suffice for the classification task. ${ }^{4}$

Finally, note that as in the original VMM, the pruning criterion defined above is not monotone. Thus, it is possible to get $I_{s_{1}}>I_{s_{2}}<I_{s_{3}}$ for $s_{3}=\operatorname{suff}\left(s_{2}\right)=\operatorname{suff}\left(\operatorname{suff}\left(s_{1}\right)\right)$. In this case we may be tempted to prune the "middle" suffix $s_{2}$ along with its child, $s_{1}$, despite the fact that $I_{s_{1}}>I_{s_{3}}$. To avoid that we define the pruning criterion more carefully. We denote by $\hat{T}_{s}$ the subtree spanned by $s$, that is, all the nodes in $\hat{T}_{s}$ correspond to subsequences with the same suffix, $s$. We can now calculate $\bar{I}_{s}=\max _{s^{\prime} \in \hat{T}_{s}} I_{s^{\prime}}$, and define the pruning criterion by $\bar{I}_{s}-I_{\text {suff }(s)} \leq \varepsilon_{2}$. Therefore, we prune $s$ (along

\footnotetext{
${ }^{4}$ Indeed, in general, conditioning reduces entropy, and therefore increases MI, but this does not say anything about the individual terms at the MI summation which may exhibit an opposite relation (cf. [6]).
}

with all its descendants), only if there is no descendant of $s$ (including $s$ itself) that induces more information (up to $\varepsilon_{2}$ ) between $\Sigma$ and $C$, compared to $\operatorname{suff}(s)$, the parent of $s$. We term this algorithm DVMM training (see Algorithm 1).

\section{SORTING THE DISCRIMINATIVE FEATURES}

The above procedure yields a rather compact discriminative model between several statistical sources. Naturally not all its features have the same discriminative power. We denote the information content of a feature by

$$
I_{\sigma \mid s} \equiv \sum_{c \in C} \hat{P}(c \mid s) \hat{P}(\sigma \mid s, c) \log \left(\frac{\hat{P}(\sigma \mid s, c)}{\hat{P}(\sigma \mid s)}\right) .
$$

Note that $I_{s}=\sum_{\sigma \in \Sigma} I_{\sigma \mid s}$, thus $I_{\sigma \mid s}$ is simply the contribution of $\sigma$ to $I_{s}$. If $\hat{P}(\sigma \mid s, C) \approx \hat{P}(\sigma \mid s)$, meaning $\sigma$ and $C$ are almost independent given $s$, then $I_{\sigma \mid s}$ will be relatively small, and vice versa.

This criterion can be applied to sort all the DVMM features. Still, it might be that $I_{\sigma_{1} \mid s_{1}}=I_{\sigma_{2} \mid s_{2}}$, while $\hat{P}\left(s_{1}\right) \gg$ $\hat{P}\left(s_{2}\right)$. Clearly in this case one should prefer the first feature, $\left\{s_{1} \cdot \sigma_{1}\right\}$, since the probability to encounter it is higher. Therefore, we should balance between $I_{\sigma \mid s}$ and $\hat{P}(s)$ when sorting. Specifically, we score each feature by $\hat{P}(s) I_{\sigma \mid s}$, and sort in decreasing order.

The pruning and sorting schemes above are based on 10 cal conditional mutual information values. We review the process from a global standpoint. The global conditional mutual information is given by (see, e.g., [6])

$$
\begin{aligned}
I(\Sigma ; C \mid S) & =\sum_{s \in \Sigma^{*}} \hat{P}(s) I(\Sigma ; C \mid s)=\sum_{s \in \Sigma^{*}} \hat{P}(s) I_{s} \\
& =\sum_{s \in \Sigma^{*}} \sum_{\sigma \in \Sigma} \hat{P}(s) I_{\sigma \mid s} .
\end{aligned}
$$

First we neglect all suffixes with a relatively small prior $\hat{P}(s)$. Then we prune all suffixes $s$ for which $\bar{I}_{s}$ is small with respect to $I_{\text {suff }(s)}$. Finally, we sort all remaining features by their 
contribution to the global conditional mutual information, given by $\hat{P}(s) I_{\sigma \mid s}$. Thus, we aim for a compact model that still strives to maximize $I(\Sigma ; C \mid S)$.

Expressing the conditional $\mathrm{MI}$ as the difference between two conditional entropies, $I(\Sigma ; C \mid S)=H(C \mid S)-H(C \mid S, \Sigma)$, we see that maximizing $I(\Sigma ; C \mid S)$ is equivalent to minimizing $H(C \mid \Sigma, S)$. In other words, our procedure effectively tries to minimize the entropy, that is, the uncertainty, over the category identity $C$ given the new symbol $\Sigma$ and the suffix $S$, which in turn decreases the classification error (see (1)).

\section{EXPERIMENTAL RESULTS}

To test the validity of our method we performed a comparative analysis over several data types. In this section, we describe the results for protein and text classification tasks. Other applications, such as DNA sequence analysis, will be presented elsewhere.

\subsection{Experimental design}

In every dataset the DVMM algorithm is compared with two different (although related) algorithms. A natural comparison is of course with the original generative VMM model [4]. In a recent work, Bejerano and Yona [19] successfully applied a one-versus-all approach to protein classification, building a generative VMM for each family, in order to estimate the membership probability of new protein to that family. Specifically, it was shown that one may accurately identify whether a protein is a member in that family or not. In our context, we build $|C|$ different generative models, one per class. A new example is then classified into the most probable class using these models. We will term this approach GVMM.

We further compared our results to A. Stolcke's perplexity pruning SRILM language modeling toolkit ${ }^{5}$ (discussed in Section 1.1). Here, again, $|C|$ generative models are trained and classification is to the most probable class. Since the SRILM toolkit is limited to 6-grams, we bounded the maximal depth of the PST's (for both DVMM and GVMM) to the equivalent suffix length 5 . For all three models, we neglected in the first step (of ignoring small $\hat{P}(s)$ ) all suffixes appearing less than twice in the training sequences. In principle, these two parameters can be fine tuned for a specific data set using standard methods, such as cross validation.

For pruning purposes we vary the analogous local decision threshold parameter in all three methods to obtain different model sizes. These are $\varepsilon_{2}, r$, and the perplexity threshold for DVMM, GVMM, and SRILM, respectively. In order to compute model sizes we sum the number of class specific features ( $s \cdot \sigma$ combinations) in each model. ${ }^{6}$

Finally, there is the issue of smoothing zero probabilities. Quite a few smoothing techniques exist, some widely

\footnotetext{
${ }^{5}$ See http://www.speech.sri.com/projects/srilm.

${ }^{6}$ For the DVMM this will be the number of retained nodes multiplied by $|\Sigma||C|$.
}

TABLE 1: Details of the protein super-family test.

\begin{tabular}{llc}
\hline Class & Protein family name & \#Proteins \\
\hline$c_{1}$ & Fungal lignin peroxidase & 29 \\
$c_{2}$ & Animal haem peroxidase & 33 \\
$c_{3}$ & Plant ascorbate peroxidase & 26 \\
$c_{4}$ & Bacterial haem catalase/peroxidase & 30 \\
$c_{5}$ & Secretory plant peroxidase & 102 \\
\hline
\end{tabular}

used by language modeling researchers (see [14] for a survey). Most of these incorporate two basic ideas: modifying the true counts of the $n$-grams to pseudo counts (which estimate expected rather than observed counts), and interpolating higher-order with lower-order $n$-gram models to compensate for under sampling. For SRILM we used a standard absolute-discounting (see [14]). The GVMM uses proportional smoothing (see [19]). For the DVMM we applied a simple plus 0.5 smoothing. ${ }^{7}$

\subsection{Protein classification tests}

The problem of automatically classifying proteins into biologically meaningful families has become very important in the last few years. For this data, obviously, there is no clear definition of higher-order features. Thus, usually each protein is represented by its ordered sequence of amino acids, resulting in a natural alphabet of all 20 different amino acids plus 3 ambiguity symbols.

There are various approaches to the classification of proteins into families, however most of these methods agree on a wide subset of the known protein world. We have chosen to compare our results to those of the PRINTS database [20] as its approach resembles ours. This database is a collection of protein family fingerprints. Each family is matched with a fingerprint of one or more short subsequences which have been iteratively refined using database scanning procedures to maximize their discrimination power in a semi-automatic procedure involving human supervision and intervention.

\subsubsection{A protein super-family test}

We first used a subset of five related protein families, all members of the Haem peroxidase super-family, taken from the PRINTS database (see Table 1 for details). Peroxidases are Haem-containing enzymes that use hydrogen peroxide as the electron acceptor to catalyse a number of oxidative reactions. We randomly chose half of the sequences as the training set and used the remaining half as test set. We repeated this process 10 times and averaged the results. For each iteration we used the training set to build the (discriminative/generative) training model(s), and then used these model(s) to classify the test sequences. DVMM and GVMM prediction were obtained using (4), where $s_{i}$ corresponds to the maximal suffix kept in the model during training.

\footnotetext{
${ }^{7}$ Notice that in all our experiments the alphabet size is fairly small (below 40). Arguably, this implies that sophisticated smoothing is less needed here, compared to large vocabularies of up to $10^{5}$ symbols (words).
} 
In Figure 2a we compare the classification accuracy of all algorithms for different model sizes (by sweeping the pruning parameter). All algorithms achieved perfect (or near perfect) classification using the minimally pruned model. However, using more intensive pruning (and hence, smaller models), DVMM consistently outperforms the other two algorithms. This is probably due to the fact that the DVMM is directly trying to minimize the discrimination error, while the other two are not. Interestingly, for the GVMM the results are not monotonic. Very small models outperform mediumsized models. This phenomenon, apparent also in the text example that follows, merits further investigation.

Equally interesting here is the list of best discriminating features. In Table 2 we present the top 10 features with respect to all suffixes of length 4 , found by the DVMM algorithm (using all the data for this run). Eight of them coincide with the fingerprints chosen by the PRINTS database to represent the respective classes. The other two short motifs which have no match in the PRINTS database are however good features as they appear in no other class but their respective one. In general these can suggest improvements for the PRINTS fingerprint, which is usually started from a manually crafted set of subsequences. It can also draw attention to conserved motifs, of possible biological importance, which a multiple alignment program (a generative method) or a human curator may have failed to notice. Finally, notice that the first seven entries in our table share but three different suffixes between them, where in each case the next symbol separates between two different classes (e.g., $R, V$ separate ARDS into classes 1 and 5, respectively. Neither appear in any of the other 4 classes). This allows to highlight polymorphisms which are family specific and thus of special interest when considering the molecular reasoning behind a biological subclassification. When a polymorphic site is not surrounded by a rather large conserved region which serves to guide a generative model such as an alignment tool or an HMM, these methods may very well fail to recognize it.

\subsubsection{A protein domain test}

As a second, harder test we used another subset of five protein groups taken from the same PRINTS database [20] (see Table 3 for details). However these five groups do not share a super-family. Rather, they all share a common domain (a domain is an independent protein structural unit). The distinction becomes clearer when we notice the members of the S-crystallin group share the same domain (and thus an evolutionary origin) with the other four groups, and yet the domain appears to perform a different function in them. In all other groups the glutathione S-transferase (GST) domain participates in the detoxification of reactive electrophilic compounds by catalysing their conjugation to glutathione. We specifically chose this test since a well-established database of protein families $\mathrm{HMMs},{ }^{8}$ currently considered the state-of-the-art in generative modeling

${ }^{8}$ The Pfam database, available at http://www.sanger.ac.uk/Pfam.

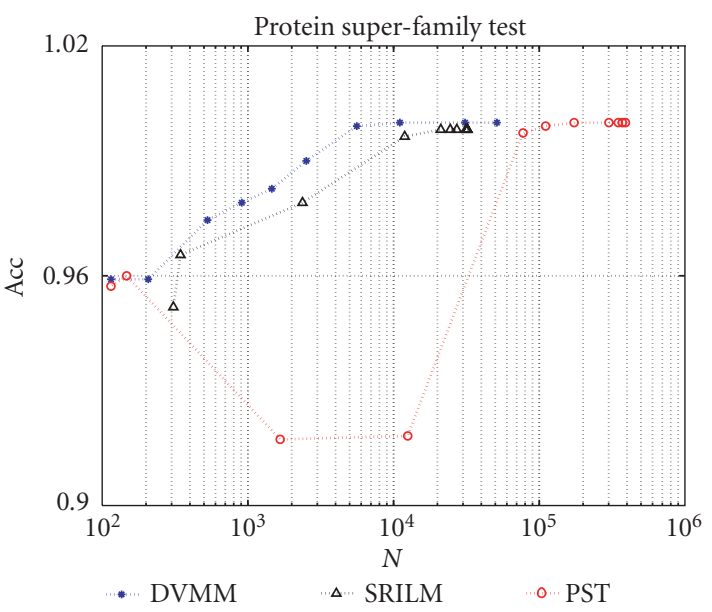

(a)

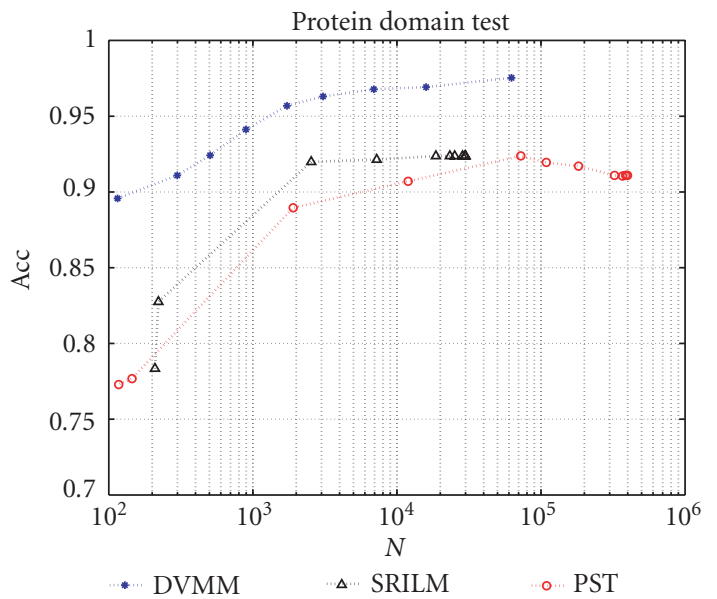

(b)

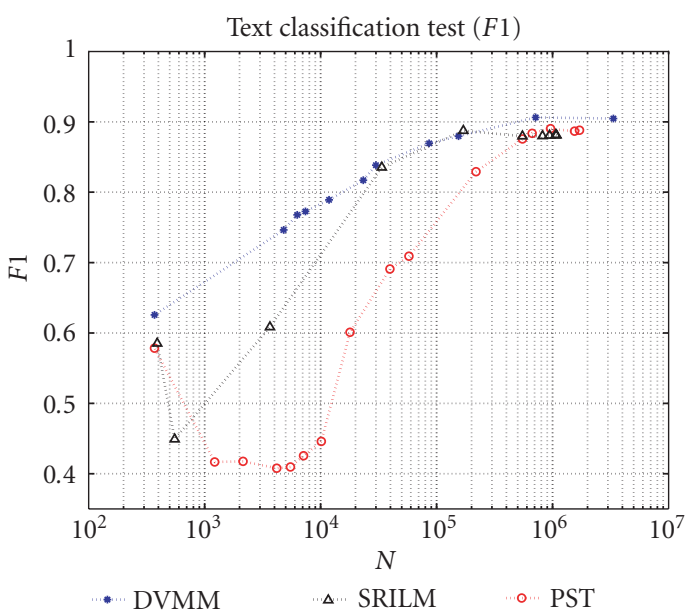

(c)

Figure 2: Comparison of the three algorithms. (a) Accuracy versus model size for all three algorithms over the protein super-family test. (b) Accuracy versus model size for all three algorithms over the protein domain test. (c) Micro-averaged F1 (see text) versus model size for all three algorithms over the text classification test. 
TABLE 2: Correlation between the top sorted features extracted by the DVMM and known motifs, for the protein super-family test. The left column presents the top 10 features among all features with memory length 4 . For example, the first feature corresponds to the suffix $s=A R D S$ followed by the symbol $R$ (the characters represent different amino acids). Additionally, the category for which $\hat{P}(\sigma \mid s, c)$ was maximized is indicated (categories are ordered as in Table 1). For the other categories, $\hat{P}\left(\sigma \mid s, c^{\prime}\right)$ was usually close to zero, and never exceeded 0.1 . Second and third columns present $\hat{P}(\sigma \mid s, c)$ and $\hat{P}(s \mid c)$ for the same (maximizing) category. The next column give the percentage of occurrences for this feature in the complete set of protein sequences in this category. The last column indicate the percentage of occurrences for this feature only in the PRINTS fingerprint of this family. For example, the feature $A R D S \mid R$ is a subsequence of a motif of the first family. It appears in this motif for $62 \%$ of the proteins assigned to it. In this table all features either came for a PRINTS motif or from elsewhere in the protein sequences (and thus the all or nothing correspondence between the last two columns).

\begin{tabular}{lcccc}
\hline Feature & $\hat{P}(\sigma \mid s, c)$ & $\hat{P}(s \mid c)$ & Sequence correlation & Fingerprint correlation \\
\hline$c_{1}: A R D S \mid R$ & 0.65 & 0.0019 & $62 \%$ & $62 \%$ \\
$c_{3}: G L L Q \mid L$ & 0.64 & 0.0029 & $73 \%$ & $73 \%$ \\
$c_{5}: A R D S \mid V$ & 0.66 & 0.0009 & $26 \%$ & $0 \%$ \\
$c_{5}: G L L Q \mid S$ & 0.38 & 0.0006 & $11 \%$ & $11 \%$ \\
$c_{3}: I V A L \mid S$ & 0.68 & 0.0035 & $88 \%$ & $88 \%$ \\
$c_{5}: G L L Q \mid T$ & 0.29 & 0.0006 & $8 \%$ & $8 \%$ \\
$c_{5}: I V A L \mid A$ & 0.28 & 0.0002 & $4 \%$ & $4 \%$ \\
$c_{4}: P W W P \mid A$ & 0.59 & 0.0008 & $64 \%$ & $64 \%$ \\
$c_{4}: A S A S \mid T$ & 0.40 & 0.0005 & $20 \%$ & $20 \%$ \\
$c_{2}: F S N L \mid S$ & 0.49 & 0.0004 & $30 \%$ & $0 \%$ \\
\hline
\end{tabular}

TABLE 3: Details of the protein domain test.

\begin{tabular}{llc}
\hline Class & Family name & \#Proteins \\
\hline$c_{1}$ & GST-no class label & 298 \\
$c_{2}$ & S crystallin & 29 \\
$c_{3}$ & Alpha class GST & 40 \\
$c_{4}$ & Mue class GST & 32 \\
$c_{5}$ & Pi class GST & 22 \\
\hline
\end{tabular}

of protein families, has chosen not to model these groups separately, due to high sequence similarity between members of the different groups. Additionally, the empirical prior probability $\hat{P}(C)$ in this test was especially skewed, since we used all GST proteins with no known subclassification as one of the groups. This is also a known difficulty for classification schemes. The experimental setting, including the parameter values, were exactly the same as for the previous test (i.e., 10 random splits into equally sized training and test set, etc.).

In spite of the above-mentioned potential pitfalls, we still found DVMM to perform surprisingly well in this test (see Figure $2 b$ ). Using the minimally pruned model, the DVMM attained almost 98\% accuracy. Moreover, for all obtained model sizes, the DVMM clearly outperformed the other two algorithms. For example, the accuracy of the DVMM while using only $\approx 500$ features was comparable to the accuracy of the GVMM while using $\approx 400,000$ features.

This relation may be explained by the high similarity between members of all classes. Since, in particular, each class displays a rich conserved structure-the GVMM concentrates on modeling this structure, disregarding the fact that it is commonly shared by all five classes. The DVMM on the other hand ignores all common statistical features, homing in only on the discriminative ones, which we know to be few in this case.

Again, in Table 4 we discuss the top 10 sorted features with respect to all suffixes of length 4 , and their correlation with known motifs.

\subsection{Text classification test}

Finally, we demonstrate the performance of the DVMM algorithm in a standard text classification task. In this experiment we set $\Sigma$ to be the set of characters present in the documents. Our pre-processing included lowering upper case characters and ignoring all non-alpha-numeric characters.

Obviously, this representation ignores the special role of the blank character as a separator between different words. Still, in many situations (as in the above protein classification task) the correct segmentation is unknown, leaving one with the basic alphabet. It should be interesting to examine text classification using the DVMM, where we take $\Sigma$ to be the set of different words that occurred in the documents. ${ }^{9}$ There we expect the DVMM to extract the most discriminant word phrases between the different categories. However, this implementation (which will probably call for sophisticated smoothing as well) is left for future research.

\footnotetext{
9 The alphabet size (and node out degree) is in general not bounded in this case. However, previous work by Pereira et al. [21] suggests practical solutions to this situation.
} 
TABLE 4: Correlation of the top sorted features extracted by the DVMM and known motifs for the protein domain test. The column headings are the same as in Table 2. Class $c_{1}$ was constructed from all GST domain-containing proteins not sharing any class specific, PRINTS or other protein database, signature. Thus they do not have a PRINTS fingerprint. Testimony of the relative difficulty of this task can be found in the fact that now only 3 of the top 10 features are unique to their class. Moreover, 6 of these features appear solely outside the PRINTS fingerprints, giving leads to a finer analysis of the GST sequences which will be done elsewhere.

\begin{tabular}{lcccc}
\hline Feature & $\hat{P}(\sigma \mid s, c)$ & $\hat{P}(s \mid c)$ & Sequence correlation & Fingerprint correlation \\
\hline$c_{3}: A A G V \mid E$ & 0.74 & 0.0037 & $77 \%$ & $52 \%$ \\
$c_{2}: A A G V \mid Q$ & 0.38 & 0.0020 & $31 \%$ & $0 \%$ \\
$c_{2}: Y I A D \mid C$ & 0.49 & 0.0016 & $34 \%$ & $31 \%$ \\
$c_{5}: L D L L \mid L$ & 0.43 & 0.0029 & $45 \%$ & $0 \%$ \\
$c_{1}: Y I A D \mid K$ & 0.46 & 0.0003 & $4 \%$ & - \\
$c_{3}: Y F P V \mid F$ & 0.42 & 0.0011 & $20 \%$ & $0 \%$ \\
$c_{2}: G R A E \mid I$ & 0.70 & 0.0043 & $93 \%$ & $0 \%$ \\
$c_{5}: D G D L \mid T$ & 0.49 & 0.0031 & $54 \%$ & $50 \%$ \\
$c_{5}: Y F P V \mid R$ & 0.45 & 0.0026 & $45 \%$ & $0 \%$ \\
$c_{5}: K E E V \mid V$ & 0.51 & 0.0029 & $54 \%$ & $0 \%$ \\
\hline
\end{tabular}

We used the standard Reuters-21578 collection. ${ }^{10}$ In particular, we took the ModeApte split and concentrated on the 10 most frequent categories. This resulted with a training set of 7194 documents and a test set of 2788 documents. We note that about $9 \%$ of these documents are multi-labeled while our implementation induces uni-labeled classification (where each document is classified only to its most probable class).

In general, we used the same parameter settings for all algorithms as in the previous section. However, to avoid exceeding memory capacity, in the first stage of the DVMM and GVMM algorithms we neglected all suffixes which appeared less than 50 times in the training set. In this setting, the run time of the DVMM (including classification) over the whole corpus was about two minutes (using a $733 \mathrm{MHz}$ PC running Linux).

In Figure 2c we present the micro-averaged $\mathrm{F} 1$ results for different model sizes for all algorithms. ${ }^{11}$ As in the previous tests, the DVMM results are consistently comparable or superior to the other algorithms. Specifically, while using the minimally pruned model, the micro-averaged precision and recall of the DVMM are $95 \%$ and $87 \%$, respectively. This implies a break-even performance of at least $87 \%$ (probably higher). We therefore compared these results with the break-even performance reported by Dumais et al. [23] for

\footnotetext{
${ }^{10}$ Available at http://www.daviddlewis.com/resources/testcollections/ reuters $21578 /$.

${ }^{11}$ The $\mathrm{F} 1$ measure is the harmonic average of the standard recall and precision measures: $F 1=2 p r /(p+r)$ (see, e.g., [22]). It is easy to verify that for a uni-labeled dataset and a uni-labeled classification scheme, the microaveraged precision and recall are equivalent, and hence equal to the F1 measure. Therefore, for the protein classification tests we simply reported the micro-averaged precision (which we termed "accuracy"). However, since the Reuters corpus is multi-labeled, our Recall performance was typically lower than our Precision.
}

the same task. In that work the authors compared five different classification algorithms: FindSim (a variant of Rocchio's method), Naive Bayes, Bayes nets, Decision Trees, and SVM. The (weighted) averaged performance of the first four were $74.3 \%, 84.8 \%, 86.2 \%$, and $88.6 \%$, respectively. The DVMM is thus superior or comparable to all these four. The only algorithm which outperforms the DVMM was the SVM with averaged performance of $92 \%$.

We see these results as especially encouraging, as all of the above algorithms were used with the words representation, while the DVMM was using the low-level character representation.

\section{DISCUSSION AND FUTURE WORK}

The main contribution of this work is in describing a welldefined framework for learning variable memory Markov models in the context of discriminative analysis. ${ }^{12}$ The DVMM algorithm enables to extract features with variable length dependencies which are highly discriminative with respect to the statistical sources at hand. These features are kept while other, possibly numerous features common to all classes, are shed. They may also gain us additional insights into the nature of the given data.

The algorithm is efficient and could be applied to any kind of data (which exhibits the Markov property), as long as a reasonable definition of (or quantization to) a basic alphabet can be derived. The method is especially appealing where no natural definition of higher level features exists, and in classification tasks where the different categories share a lot of structure (which generative models will capture, in vain).

Several important directions are left for future work. On

\footnotetext{
${ }^{12}$ For a related approach to discrimination, using competitive learning of generative PSTs see [24].
} 
the empirical side, more extensive experiments are required. For the protein data, a thorough analysis of the top discriminating features and their possible biological function is appealing.

On the theoretical aspect, a formal analysis of the algorithm is missing. It may even be possible to extend the theoretical results presented in [4], in the context of discriminative VMM models.

\section{ACKNOWLEDGMENTS}

Useful discussion with Y. Bilu, R. Bachrach, E. Schneidman, and $\mathrm{E}$. Shamir is greatly appreciated. The authors would also like to thank A. Stolcke for his help in using the SRILM toolkit.

\section{REFERENCES}

[1] H. Almuallim and T. G. Dietterich, "Learning with many irrelevant features," in Proc. 9th National Conference on Artificial Intelligence (AAAI '91), vol. 2, pp. 547-552, Anaheim, Calif, USA, July 1991.

[2] G. F. Hughes, "On the mean accuracy of statistical pattern recognizers," IEEE Trans. on Information Theory, vol. 14, no. 1, pp. 55-63, 1968.

[3] E. B. Baum and D. Haussler, "What size net gives valid generalization?," Neural Computation, vol. 1, no. 1, pp. 151-160, 1989.

[4] D. Ron, Y. Singer, and N. Tishby, "The power of amnesia: learning probabilistic automata with variable memory length," Machine Learning, vol. 25, pp. 237-262, 1997.

[5] P. M. Lewis, "The characteristic selection problem in recognition systems," IRE Trans. on Information Theory, vol. 8, no. 2, pp. 171-178, 1962.

[6] T. M. Cover and J. A. Thomas, Elements of Information Theory, John Wiley \& Sons, New York, NY, USA, 1991.

[7] R. M. Goodman and P. Smyth, "Decision tree design from communication theory stand point," IEEE Trans. on Information Theory, vol. 34, no. 5, pp. 979-994, 1988.

[8] R. Battiti, "Using mutual information for selecting features in supervised neural net learning," IEEE Transactions on Neural Networks, vol. 5, no. 4, pp. 537-550, 1994.

[9] G. Barrows and J. Sciortino, "A mutual information measure for feature selection with application to pulse classification," in IEEE Intern. Symposium on Time-Frequency and Time-Scale Analysis, pp. 249-253, 1996.

[10] H. Yang and J. Moody, "Feature selection based on joint mutual information," in Proc. International ICSC Symposium on Advances in Intelligent Data Analysis, Rochester, NY, USA, June 1999.

[11] K. Torkkola and W. M. Campbell, "Mutual information in learning feature transformation," in Proc. 17th International Conference on Machine Learning (ICML '2000), pp. 10151022, Stanford, Calif, USA, 2000.

[12] S. Della Pietra, V. Della Pietra, and J. Lafferty, "Inducing features of random fields," IEEE Trans. on Pattern Analysis and Machine Intelligence, vol. 19, no. 4, pp. 380-393, 1997.

[13] A. K. McCallum, Reinforcement learning with selective perception and hidden states, Ph.D. thesis, University of Rochester, Rochester, NY, USA, 1996.

[14] S. F. Chen and J. T. Goodman, "An empirical study of smoothing techniques for language modeling," Tech. Rep. TR-10-98, Harvard University, Mass, USA, August 1998.
[15] A. Stolcke, "Entropy-based pruning of backoff language models," in Proc. DARPA Broadcast News Transcription and Understanding Workshop, pp. 270-274, Lansdowne, Va, USA, February 1998.

[16] L. R. Bahl, P. F. Brown, P. V. de Souza, and R. L. Mercer, "Maximum mutual information estimation of hidden Markov model parameters for speech recognition," in Proc. IEEE Int. Conf. Acoustics, Speech, Signal Processing, vol. 1, pp. 49-52, Tokyo, Japan, October 1986.

[17] Y. Normandin, R. Cardin, and R. De Mori, "Highperformance connected digit recognition using maximum mutual information estimation," IEEE Trans. Speech, and Audio Processing, vol. 2, no. 2, pp. 299-311, 1994.

[18] P. C. Woodland and D. Povey, "Large scale discriminative training for speech recognition," in Proc. Int. Workshop on Automatic Speech Recognition (ASR), pp. 7-16, Paris, France, September 2000.

[19] G. Bejerano and G. Yona, "Variations on probabilistic suffix trees: statistical modeling and prediction of protein families," Bioinformatics, vol. 17, no. 1, pp. 23-43, 2001.

[20] T. K. Attwood, M. J. Blythe, D. R. Flower, et al., "PRINTS and PRINTS-S shed light on protein ancestry," Nucleic Acids Res., vol. 30, no. 1, pp. 239-241, 2002.

[21] F. Pereira, Y. Singer, and N. Tishby, "Beyond word N-grams," in Natural Language Processing Using Very Large Corpora, K. Church, S. Armstrong, P. Isabelle, E. Tzoukermann, and D. Yarowsky, Eds., pp. 95-106, Kluwer Academic, Dordrecht, 1999.

[22] Y. Yang, "A study on thresholding strategies for text categorization," in Proc. 24th Annual International ACM SIGIR Conference on Research and Development in Information Retrieval, pp. 137-145, New Orleans, La, USA, September 2001.

[23] S. Dumais, J. Platt, D. Heckerman, and M. Sahami, "Inductive learning algorithms and representations for text categorization," in Proc. International Conference on Information and Knowledge Management, pp. 148-155, Bethesda, Md, USA, November 1998.

[24] G. Bejerano, Y. Seldin, H. Margalit, and N. Tishby, "Markovian domain fingerprinting: statistical segmentation of protein sequences," Bioinformatics, vol. 17, no. 10, pp. 927-934, 2001.

Noam Slonim received his B.S. degree in computer science, physics, and mathematics in 1995 from the Hebrew University of Jerusalem, Israel. He submitted his Ph.D. thesis, entitled "The Information Bottleneck: Theory and Applications" at the Hebrew University in 2002. He is currently a Research Associate with the Biophysics Group of the Princeton University Physics Department. The primary aim of his research is the development of new, theoretically well founded methods for complex data analysis. In particular he is interested in methods that are based on information theory and statistical learning theory.

Gill Bejerano received his B.S. degree in mathematics, physics, and computer science in 1997, Summa cum Laude. To date he is a Ph.D. student at the School of Computer Science and Engineering, in the Hebrew University. His primary research interests are computational molecular biology and bioinformatics, computational and statistical learning theory and information theory. Gill Bejerano is a recent recipient of a Best Paper by a Student Award, and a Best Poster Award. 
Shai Fine received his Ph.D. degree in computer science from the Hebrew University in 1999. Since then he has been a research member at IBM Research Labs. His primary research interests are machine learning problems that arise in human-machine interaction and incorporate temporal modeling, statistical inference, and discriminative methods. Related applications are the modeling of text, natural language understanding, speaker and speech recognition. More recently he has been working on applying machine learning techniques to problems in simulation-based functional verification of hardware design.

Naftali Tishby is currently the Chair of the Computer Engineering Program at the School of Computer Science and Engineering and a member of the Interdisciplinary Center for Neural Computation at the Hebrew University. He received his Ph.D. degree in theoretical physics from the Hebrew University in 1985 and has been a research member of staff at MIT, Bell Labs, AT\&T, and NECI since then. His current research is on the interface between computer science, statistical physics, and computational biology. He introduced various methods from statistical mechanics into computational learning theory and machine learning. More recently, he has been working on the foundation of biological information processing and has developed novel learning algorithms based on information theory, such as the Information Bottleneck method and Sufficient Dimensionality Reduction. 